\title{
Факторы риска развития и проявления острого холангита у больных с доброкачественной обструкцией внепеченочных желчных путей
}

\author{
И. Н. Мамонтов, Т. И. Тамм, К. А. Крамаренко, С. Г. Белов, А. Я. Бардюк,
} А. П. Захарчук, В. В. Непомнящий, Е. А. Шакалова, О. М. Решетняк

Харьковская медицинская академия последипломного образования

\section{The risk factors for development and demonstration of an acute cholangitis in patients, suffering benign obstruction of extrahepatic biliary ducts}

\author{
I. N. Mamontov, T. I. Tamm, K. A. Kramarenko, S. G. Belov, A. Ya. Bardyuk, \\ A. P. Zakharchuk, V. V. Nepomnyashchiy, E. A. Shakalova, O. M. Reshetnyak \\ Kharkov Medical Academy of Postgraduate Education
}

\section{Реферат}

Цель. Определение факторов, способствующих развитию острого холангита (OX) или являющихся его признаками при доброкачественной обструкции внепеченочных желчных путей (ДОВЖП).

Материалы и методы. Ретроспективно проанализированы 144 наблюдения ДОВЖП, ОХ отмечен в 17 наблюдениях Проведено сравнение встречаемости различных факторов и признаков у больных с ОХ и без ОХ.

Результаты. Различия выявлены ( < 0,05) между такими показателями: гипертермия, триада Шарко, лейкоцитоз, содержание палочкоядерных нейтрофилов, уровень общего билирубина, активность амилазы крови, толщина стенки желчного пузыря (ЖП), наличие отключенного ЖП, фиксированного камня большого сосочка двенадцатиперстной кишки (БСДПК), размер БСДПК. Различий не обнаружено (p > 0,05) между следующими показателями: пол, возраст, индекс массы тела, наличие абдоминального болевого синдрома, холецистэктомия в анамнезе, объем ЖП, причина обструкции, расширение гепатикохоледоха, эндоскопическая папиллосфинктеротомия в анамнезе, наличие околососочкового дивертикула и острого билиарного панкреатита.

Выводы. Факторы риска развития ОХ: уровень общего билирубина 70 мкмоль/л и выше, утолщение стенки жП до 4 мм и более, отключенный ЖП, фиксированный камень БСДПК, размер БСДПК 15 мм и более. Проявления ОХ: гипертермия, наличие триады Шарко, лейкоцитоз $9 \times 10^{9}$ в 1 л и выше, увеличение содержания палочкоядерных нейтрофилов до 7\% и выше, гиперамилаземия.

Ключевые слова: острый холангит; факторы риска; проявления; обструкция желчных путей.

Abstract

Objective. Determination of factors, promoting development of an acute cholangitis (ACH) or constituting its signs in benign obstruction of extrahepatic biliary ducts (EHBD).

Materials and methods. Retrospectively 144 observations of benign obstruction of extrahepatic biliary ducts, ACH was noted in 17 observations. Comparison of prevalence of various factors and signs in the patients with ACH and without it was accomplished. Results. The difference was statistically proven $(\mathrm{p}<0.05)$ between such indices: hyperthermy, Charcot's triad, leucocytosis, content of stab neutrophils, level of general bilirubin, the blood amylase activity, the gallbladder wall thickness, presence of excluded gallbladder, fixated calculus of duodenal papilla magna (DPM), dimensions of DPM. The differences were absent $(p>0.05)$ between following indices: gender, age, BMI, presence of abdominal pain syndrome, cholecystectomy in anamnesis, the gallbladder volume, cause of obstruction, dilation of hepaticocholedochus, endoscopic papillosphincterotomy in anamnesis, presence of peripapillary diverticulum and an acute biliary pancreatitis.

Conclusion. The risk factors for development of ACH were: a general bilirubin level $70 \mathrm{mcmol} / \mathrm{l}$ and higher, the gallbladder wall thickening up to $4 \mathrm{~mm}$ and more, the excluded gallbladder, fixated calculus of DPM, dimension of DPM $15 \mathrm{~mm}$ and more. Signs of an ACH were: hyperthermy, presence of Charcot's triad, leucocytosis $9 \times 10^{9}$ in 11 and higher, enhancement of content of stab neutrophils up to $7 \%$ and higher, hyperamylasemia.

Keywords: acute cholangitis; the risk factors; signs; obstruction of biliary ducts.

Острый холангит (OX) - осложнение билиарной обструкции, характеризующееся присоединением бактериальной инфекции и развитием воспаления желчных протоков. Наиболее частой причиной холестаза при развитии ОХ является холедохолитиаз [1 - 4]. Основными путями инфицирования желчи считают: восходящий - из двенадцатиперстной кишки (ДПК), гематогенный - через венозную кровь портальной системы и инструментальный - после удаления камней, установки стентов и др. [3, 4]. Исследования, посвященные изучению факторов, предрасполагающих к развитию ОХ, немногочисленны и противоречивы [5-9]. 
Диагностика холангита основывается на выявлении обструкции желчных протоков в сочетании с клиническими признаками и данными лабораторных и инструментальных методов исследования $[2,10]$.

Клиническими признаками являются гипертермия и/ или потрясающий озноб, желтуха и выраженная боль в верхних правых отделах живота. Триада Шарко - сочетание гипертермии, абдоминального болевого синдрома и желтухи - специфична для больных с холангитом, однако имеет низкую чувствительность - 26,4\% [10].

Среди лабораторных признаков учитывают гипербилирубинемию, изменения белой крови, характерные для системного воспалительного ответа, повышение активности печеночных проб (щелочная фосфатаза, гамма-глютамилтранспептидаза, аланинаминотрансфераза, аспартатаминотрансфераза) в 1,5 раза и более в сравнении с верхней границей нормы и некоторые другие показатели (С-реактивный белок) [10].

Инструментальная диагностика направлена на выявление самого факта обструкции внепеченочных желчных путей (ВЖП), включающее ультразвуковое исследование (УЗИ), компьютерную (КТ) и магнитно-резонансную (МРТ) томографию, эндоскопическую ретроградную холангиопанкреатографию (ЭРХПГ), косвенных (КТ, МРТ) и прямых признаков (гнойная желчь) холангита [10].

Течение ОХ может варьировать от легкого до тяжелого с развитием сепсиса, сопровождающегося синдромом полиорганной недостаточности, смертность при котором достигает 5 - 10\% [10, 11].

Первичное лечение ОХ включает антибактериальную терапию и выполнение как можно раньше билиарной декомпрессии. Используемые для этого ЭРХПГ и сопутствующие ей эндоскопические вмешательства являются эффективными и безопасными в восстановлении желчеоттока $[2,11]$.

Выявление факторов риска развития ОХ и его признаков у больных с доброкачественной обструкцией внепеченочных желчных путей (ДОВЖП) позволит уточнить патогенетические особенности развития этого осложнения, выделить больных, предрасположенных к развитию OX, и/или провести его раннюю диагностику.

Цель исследования: определение факторов, способствующих развитию ОХ или являющихся его признаками у больных с ДОВЖП.

\section{Материалы и методы исследования}

За период с января 2014 по декабрь 2016 г. проведен ретроспективный анализ историй болезни 215 больных с обструкцией ВЖП, находившихся на лечении в клинике кафедры хирургии и проктологии Харьковской медицинской академии последипломного образования на базе хирургических отделений Харьковской городской клинической больницы № 2 имени проф. А. А. Шалимова.

Алгоритм диагностики и лечения включал сбор анамнеза, лабораторные исследования крови, абдоминальное УЗИ, ЭРХПГ с эндоскопическими вмешательствами, направленными на восстановление желчеоттока. При подозрении на опухолевый характер обструкции и в диагностически неясных случаях выполняли КТ с внутривенным контрастированием. Проводимая консервативная терапия была направлена на стабилизацию и поддержание основных жизненных функций, а также профилактику и лечение осложнений. При необходимости проводили оперативное лечение.

Критерием обструкции ВЖП было расширение гепатикохоледоха до 8 и более мм по данным УЗИ или другого визуализирующего метода.

Из исследования исключены 36 пациентов с опухолевой обструкцией и 4 пациента, у которых причиной обструкции был хронический панкреатит, ввиду особенностей течения основного заболевания, а также из-за их малого количества и отсутствия у них холангита.

Из 175 больных с ДОВЖП в исследование включены 144. Основным критерием включения больных в исследование была ЭРХПГ с эндоскопическими вмешательствами, выполненная не позднее 32 ч после госпитализации, то есть в день поступления либо на следующий день. Пациентов, которым ЭРХПГ выполняли позже указанного временного промежутка, не включали в исследование, так как проводимая в течение этого времени консервативная терапия, в том числе и антибактериальная, могла повлиять на клинические и эндоскопические проявления заболевания и способствовать ложноотрицательному диагнозу холангита у этих пациентов.

Основным критерием ОХ была гнойная желчь, выявленная при ЭРХПГ.

С целью определения факторов, способствующих развитию ОХ или являющихся его проявлениями, изучали пол, возраст и индекс массы тела (ИМТ) больных; клинические признаки - наличие абдоминального болевого синдрома, гипертермии, триады Шарко; лабораторные данные - количество лейкоцитов и палочкоядерных нейтрофилов, уровень билирубина и активность амилазы крови; состояние желчного пузыря (ЖП) - его наличие (холецистэктомия в анамнезе), объем, толщина стенки, наличие отключенного ЖП (заполненный камнями, несодержащий желчи); состояние ВЖП - диаметр гепатикохоледоха, эндоскопическая папиллосфинктеротомия (ЭПСТ) в анамнезе; причины обструкции ВЖП - наличие холедохолитиаза и его вид: одиночный (1 - 2 камня), множественный (3 и более камней) холедохолитиаз, сладж общего желчного протока (ОЖП), синдром Мириззи, наличие фиксированного камня и стеноза большого сосочка двенадцатиперстной кишки (БСДПК); наличие околососочкового дивертикула, который может быть отнесен как к факторам состояния ВЖП, так и к причинам нарушения оттока желчи; размер БСДПК.

Объем и толщину стенки ЖП, отключенный жП и диаметр гепатикохоледоха оценивали по результатам УЗИ; причину обструкции, наличие околососочкового дивертикула, размер БСДПК - на основании данных ЭРХПГ и эндоскопических вмешательств. 
Количественные признаки разбивали на градации, качественные оценивали по их наличию или отсутствию. Статистическая обработка каждого признака проведена с использованием критерия $\chi^{2}$ Пирсона. Достоверными считали различия, если величина р была меньше 0,05.

\section{Результаты}

Из 144 больных, включенных в данное аналитическое исследование, острый гнойный холангит отмечен у 17 (11,8\%). У 127 (88,2\%) пациентов с ДОВжП не наблюдали клинических и эндоскопических проявлений холангита. Никто из пациентов не умер.

Из данных табл. 1 видно, что пол, возраст и ИМТ больных не оказывают существенного влияния на развитие ОХ при ДОВжП (p > 0,05). Абдоминальный болевой син- дром отмечали одинаково часто (p > 0,05) как у больных с ДОВЖП и ОХ, так и у больных с ДОВЖП без ОХ. Характерной для ОХ была гипертермия, которую наблюдали почти в 5 раз чаще, чем у больных без холангита ( $<0,001)$. Триада Шарко, специфичный признак для холангита, выявлена только у 23,5\% больных с ОХ. Тем не менее, это в 7,3 раза чаще, чем у больных без холангита $(\mathrm{p}<0,001)$.

Анализ показателей крови позволил установить, что для больных с ОХ специфичным является лейкоцитоз 9 × $10^{9}$ в 1 л, выявленный у 52,9\% больных. У больных без холангита такой уровень лейкоцитов зафиксировали в 1,8 раза реже - у 27,6\% больных ( $<<0,05)$. Также характерным для ОХ было увеличение содержания палочкоядерных нейтрофилов до 7\% и выше. Его выявляли у этих больных в 2,8 раза чаще, чем у больных без холангита -

\begin{tabular}{|c|c|c|c|c|c|c|}
\hline \multirow{3}{*}{ Показатель } & \multicolumn{4}{|c|}{ Группа больных } & \multicolumn{2}{|c|}{ Статистические показатели } \\
\hline & \multicolumn{2}{|c|}{$\begin{array}{c}c \text { OX } \\
(n=17)\end{array}$} & \multicolumn{2}{|c|}{$\begin{array}{l}\text { без оX } \\
(n=127)\end{array}$} & \multirow[t]{2}{*}{$x^{2}$} & \multirow[t]{2}{*}{$\mathrm{p}$} \\
\hline & абс. & $\%$ & абс. & $\%$ & & \\
\hline \multicolumn{7}{|l|}{ Характеристика пациентов } \\
\hline \multicolumn{7}{|l|}{ Пол } \\
\hline M & 4 & 24,5 & 35 & 27,6 & \multirow{2}{*}{0,123} & \multirow{2}{*}{0,726} \\
\hline ж & 13 & 75,5 & 92 & 72,4 & & \\
\hline возраст 70 лет и старше & 8 & 47,6 & 44 & 34,6 & 1,001 & 0,465 \\
\hline ИМТ более $35 \mathrm{kr} / \mathrm{m}^{2}$ & 4 & 23,5 & 13 & 10,2 & 2,545 & 0,111 \\
\hline \multicolumn{7}{|l|}{ Клинические проявления } \\
\hline абдоминальный болевой синдром & 16 & 94,1 & 110 & 86,6 & 0,772 & 0,380 \\
\hline гипертермия $37,3^{\circ} \mathrm{C}$ и выше & 12 & 70,6 & 8 & 6,3 & 51,813 & $<0,001$ \\
\hline триада Шарко & 4 & 23,5 & 4 & 3,2 & 11,868 & $<0,001$ \\
\hline \multicolumn{7}{|l|}{ Лабораторные данные } \\
\hline лейкоцитоз $9 \times 10^{y}$ в 1 л & 9 & 52,9 & 35 & 27,6 & 4,552 & 0,033 \\
\hline $\begin{array}{l}\text { количество палочкоядерных } \\
\text { нейтрофилов 7\% и выше }\end{array}$ & 13 & 76,5 & 35 & 27,6 & 16,141 & $<0,001$ \\
\hline $\begin{array}{l}\text { уровень общего билирубина } \\
70 \text { мкмоль/л и выше }\end{array}$ & 13 & 76,5 & 39 & 30,7 & 13,609 & $<0,001$ \\
\hline активность амилазы выше 32 г × ч/л & 7 & 41,2 & 21 & 16,5 & 5,812 & 0,016 \\
\hline \multicolumn{7}{|l|}{ Состояние ЖП } \\
\hline холецистэктомия в анамнезе & 2 & 11,8 & 27 & 21,3 & 0,840 & 0,360 \\
\hline объем ЖП 50 см³ и выше* & 7 & 46,7 & 36 & 36 & 0,634 & 0,426 \\
\hline толщина стенки ЖП 4 мм и выше* & 7 & 46,7 & 10 & 10 & 13,921 & $<0,001$ \\
\hline отключенный жп* & 3 & 20 & 5 & 5 & 4,534 & 0,034 \\
\hline \multicolumn{7}{|l|}{ Причина обструкции, состояние ВЖП } \\
\hline Холедохолитиаз любой & 15 & 88,2 & 93 & 73,2 & 1,801 & 0,180 \\
\hline одиночный (1 - 2 камня) & 8 & 47,1 & 59 & 46,5 & 0,002 & 0,963 \\
\hline множественный (3 камня и более) & 4 & 23,5 & 20 & 15,7 & 0,654 & 0,419 \\
\hline Синдром Mirizzi & 1 & 5,9 & 2 & 1,6 & 1,364 & 0,243 \\
\hline Сладж Ожп & 2 & 11,8 & 13 & 10,2 & 0,038 & 0,847 \\
\hline Фиксированный камень БСДПК & 2 & 11,8 & 3 & 2,4 & 3,955 & 0,047 \\
\hline Стеноз БСДПК & 2 & 11,8 & 29 & 22,8 & 1,088 & 0,298 \\
\hline Диаметр гепатикохоледох 12 мм и более & 11 & 74,7 & 95 & 74,8 & 0,787 & 0,376 \\
\hline ЭПСТ в анамнезе & 2 & 11,8 & 7 & 5,5 & 1,000 & 0,318 \\
\hline Околососочковый дивертикул & 4 & 23,5 & 30 & 23,6 & 0,000 & 0,994 \\
\hline Размер БСдПк 15 мм и более & 10 & 58,8 & 39 & 30,7 & 5,279 & 0,022 \\
\hline Острый билиарный панкреатит & 4 & 23,5 & 15 & 11,8 & 1,798 & 0,181 \\
\hline Примечание. $\quad$ * - Данные без учета больн & $\mathrm{let}$ & ией в & & & & \\
\hline
\end{tabular}


соответственно у 76,5 и 27,6\% больных (p < 0,001). Уровень билирубина в крови 70 мкмоль/л и выше зафиксирован у 76,5\% больных с ОХ, что было в 2,5 раза чаще, чем у больных без ОХ - 30,7\% (p < 0,001). Нормальное значение активности амилазы ( 32 г × ч/л и ниже) относительно специфично для больных с ДОВжП без холангита, так как его определяли у $83,5 \%$ пациентов, что в 1,4 раза чаще, чем у больных с холангитом ( $<<0,05)$. Гиперамилаземия (выше 32 г × ч/л) была характерна для ОХ, ее наблюдали у $41,2 \%$ больных, что в 2,5 раза чаще, чем у больных без холангита $-16,5 \%(\mathrm{p}<0,05)$.

Проанализировав данные о состоянии ЖП, мы установили, что холецистэктомия в анамнезе и объем ЖП не влияют на развитие OX (p > 0,05). Группы больных достоверно различались по толщине стенки ЖП и наличию отключенного ЖП. Так, толщину стенки ЖП 4 мм и больше у больных с ДОВжП и холангитом отмечали в 4,7 раза чаще, чем у больных с ДОВЖП без холангита (p < 0,001), а отключенный ЖП наблюдали в 3,75 раза чаще при ОХ, нежели без него $(\mathrm{p}<0,05)$.

Не выявлено достоверной связи между причинами обструкции и частотой развития ОХ. Ни наличие камней, ни вид холедохолитиаза не влияют на присоединение OX у больных с ДОВЖП (p > 0,05). Также не выявлено влияния стеноза БСДПК на частоту развития холангита (p > 0,05). Фиксированный камень БСДПК выявляли в 5 раз чаще при $\mathrm{OX}(\mathrm{p}<0,05)$. Диаметр гепатикохоледоха, ЭПСТ в анамнезе и околососочковый дивертикул не влияют на частоту развития ОХ у больных с ДОВЖП (р > 0,05).

Достоверно различались показатели размера БСДПК. Так, БСДПК 15 мм и более наблюдали у 58,8\% больных с холангитом, что почти в 2 раза чаще, чем у больных без холангита $-30,7 \%(\mathrm{p}<0,05)$.

Наличие острого билиарного панкреатита у больных с ДОВЖП не оказывало достоверного влияния на частоту развития холангита $(\mathrm{p}>0,05)$.

\section{Обсуждение}

В нашем исследовании у больных с ДОВЖП мы проанализировали встречаемость факторов, которые потенциально могут влиять на развитие OX, и частоту признаков ОХ. Проведенный анализ показал отсутствие влияния возрастного и гендерного показателя на развитие OX у больных с ДОВЖП. В аналогичных исследованиях [5 - 9] также не выявлено какой-либо взаимосвязи между полом больных и развитием у них ОХ. Однако по данным других исследований [6 - 9] частота развития холангита возрастает с увеличением возраста пациентов. По нашим данным ОХ развивается одинаково часто у больных как старше, так и моложе 70 лет ( $>$ > 0,05).

Изучив ряд исследований [5 - 9], мы не обнаружили анализа влияния ИМТ на частоту развития ОХ. Полученные нами результаты подтверждают, что этот показатель не влияет на частоту развития OX.

В отношении клинических проявлений (абдоминальный болевой синдром, гипертермия, триада Шарко) по- лученные нами данные коррелируют с данными других исследований $[9,10]$, указывая на высокую специфичность $(96,9 \%)$ и низкую чувствительность $(23,5 \%)$ триады Шарко и на гипертермию как характерный признак для больных с ОХ, являющуюся одним из проявлений системного воспалительного ответа [10]. Необходимо отметить, что болевой синдром как один из элементов триады Шарко мы учитывали только при его локализации в правом подреберье и если он был интенсивный. Невыраженная боль другой локализации не учитывалась нами как элемент триады Шарко. Этим объясняется разница в нашем исследовании между больными с OХ и больными без ОХ по встречаемости триады Шарко (p < 0,001) и абдоминального болевого синдрома ( $\mathrm{p}>0,05)$.

Проявлениями системного воспалительного ответа являются также изменения белой крови, отражающие инфекционный процесс в желчных путях у больных с OX [10]. Это подтверждено и результатами нашего исследования - определены достоверные различия между больными с ОХ и больными без ОХ в отношении лейкоцитоза $9 \times 10^{9}$ в 1 л и выше $(\mathrm{p}<0,05)$ и количества палочкоядерных нейтрофилов 7\% и выше ( $\mathrm{p}<0,001)$.

Полученные нами данные показывают, что гипербилирубинемия 70 мкмоль/л и выше более характерна для $\mathrm{OX}$ $(\mathrm{p}<0,001)$. В других исследованиях в отношении содержания общего билирубина у больных с холангитом результаты противоречивы. В одних исследованиях не выявлено корреляции между гипербилирубинемией и наличием холангита [7, 9], а в других, напротив, у больных с холангитом определяли более высокое содержание билирубина [6,8]. Утверждается даже, что уровень билирубина может выступать прогностическим признаком тяжести холангита [8] или смерти больного [5]. По нашему мнению, гипербилирубинемия отражает степень нарушения оттока желчи наблюдаемый в нашем исследовании более высокий уровень общего билирубина у больных с ОХ соответствовал более выраженной и/или длительной обструкции. То есть фактором, предрасполагающим к развитию ОХ, является не содержание билирубина 70 мкмоль/л и выше, а обструкция желчных протоков, которая проявляется более высоким уровнем общего билирубина крови.

Показатель активности амилазы крови обычно детально не рассматривают при изучении холангита в связи с отсутствием корреляции [9]. Нами выявлена такая связь - гиперамилаземия (более 32 г × ч/л) встречалась достоверно чаще у больных с OX (p < 0,05). Это можно объяснить, с одной стороны, значительным числом больных с острым билиарным панкреатитом и ОХ (4 из 17), а с другой - гиперамилаземия при ОХ может носить неспецифический характер. В то же время, проанализировав частоту острого билиарного панкреатита у больных с ОХ и без него, мы не выявили достоверной связи (р>0,05). Из этого следует, что у больных с ДОВжП острый билиарный панкреатит не способствует развитию OX.

Нами выявлена связь между состоянием ЖП и развитием ОХ. Так, толщину стенки ЖП 4 мм и более и наличие 


\begin{tabular}{|c|c|c|c|c|c|c|}
\hline \multicolumn{7}{|c|}{$\begin{array}{l}\text { Распределение больных с ДОВжП в зависимости от вариантов сочетани у них ОХ, острого } \\
\text { и хронического холецистита }\end{array}$} \\
\hline \multirow{3}{*}{ Наличие или отсутствие воспаления ЖП } & \multicolumn{4}{|c|}{ довжп } & \multirow{3}{*}{$x^{2}$} & \multirow{3}{*}{$p$} \\
\hline & \multicolumn{2}{|c|}{$\begin{array}{c}c \text { OX } \\
(n=15)\end{array}$} & \multicolumn{2}{|c|}{$\begin{array}{c}\text { без OX } \\
(n=100)^{*}\end{array}$} & & \\
\hline & абс. & $\%$ & абс. & $\%$ & & \\
\hline Острый холецистит & 4 & 26,7 & 6 & 6 & & \\
\hline Хронический холецистит & 5 & 33,3 & 9 & 9 & 16,089 & $<0,001$ \\
\hline Без холецистита & 6 & 40 & 85 & 85 & & \\
\hline
\end{tabular}

отключенного ЖП достоверно чаще наблюдали у больных с OX ( $p<0,001$ и 0,05 соответственно). Утолщение стенки ЖП свидетельствует об остром или хроническом его воспалении. Отключенный ЖП также является проявлением длительного хронического холецистита. Достоверно большее количество пациентов с ОХ, у которых ЖП был с признаками воспаления - острого или хронического (толщина стенки 4 и более мм, отключенный ЖП), свидетельствует о том, что воспаленный ЖП способствует развитию холангита. Возможным объяснением этому является то, что воспаленный ЖП выступает источником инфицирования желчи при ДОВжП.

Наряду с этим показатели объема ЖП у больных с ДОВЖП и ОХ и без ОХ не различались. Так как для острого холецистита характерно увеличение объема ЖП, наличие различий в исследуемых группах больных по показателю толщины стенки ЖП и их отсутствие по показателю объема ЖП позволяют сделать заключение, что острота воспалительного процесса не влияет на развитие OX сам факт воспаления ЖП как острого, так и хронического является одним из факторов у больных с ДОВЖП, приводящих к развитию ОХ.

В аналитических работах, посвященных факторам развития холангита, рассмотренные нами признаки воспаления жП не изучались [5 - 9]. Только в двух исследованиях $[6,8]$ проанализировали сам факт наличия ЖП (холецистэктомия в анамнезе), по которому между группами пациентов с холангитом и без него не выявлено достоверных различий. В нашем исследовании такие различия также отсутствовали (p > 0,05). То есть у больных с холестазом не факт наличия ЖП предрасполагает к развитию холангита, а, как указывалось выше, его воспаление.

На основании признаков острого и хронического холецистита (толщина стенки и объем ЖП, наличие отключенного ЖП) мы сравнили встречаемость признаков холецистита в группах больных с ОХ и без него (табл. 2) и выявили наличие высокодостоверной связи $\left(\chi^{2}=16,089 ; \mathrm{p}\right.$ $<0,001)$. Это подтверждает, что у больных с ДОВжП воспаленный ЖП способствует развитию ОХ.

Для более глубокого анализа выявленной связи между наличием холецистита и развитием OХ у больных с ДОВжП необходимо сопоставление результатов бактериологического исследования желчи из просвета ЖП и гепатикохоледоха.
При исследовании причин, вызвавших ДОВжП, не выявлено закономерности в отношении ОХ. Это касается как наличия холедохолитиаза, так и его вида (одиночного или множественного), сладжа, а также стеноза БСДПК - различия между перечисленными показателями у больных с OX и без ОХ недостоверны ( $>$ > ,05). Это согласуется с результатами других исследований, в которых также не выявлено закономерной связи между наличием, видом холедохолитиаза и развитием холангита $[6,7]$. Из этого следует, что вид холедохолитиаза не оказывает влияния на развитие холангита. Определяющей является степень обструкции, о чем, как указывалось выше, свидетельствует достоверно большие значения билирубина у больных с OX.

В отношении околососочкового дивертикула результаты исследований противоречивы: одни авторы [7, 8] расценивают его наличие как фактор риска развития холангита, другие достоверной связи не выявили [9]. Согласно полученным нами результатам околососочковый дивертикул встречается одинаково часто у больных с холангитом и без него ( $>$ > 0,05), не являясь фактором риска развития OX.

Отсутствует также связь между ОХ и степенью расширения гепатикохоледоха (p > 0,05). Это согласуется с результатами других исследований [7 - 9].

В ряде работ [5 - 9] нет сведений о перенесенной в прошлом ЭПСТ у больных с холангитом и без него. В нашем исследовании не выявлено различий между группами больных с ОХ и без него в зависимости от наличия ЭПСТ в анамнезе $(\mathrm{p}>0,05)$.

С другой стороны, изменения БСДПК, а именно его размер 15 мм и более, и наличие фиксированного камня БСДПК существенно влияют на развитие ОХ - у больных с ОХ эти признаки встречаются достоверно чаще (р $<0,05)$. Хотя и существует связь между этими признаками (при фиксированном камне БСДПК его размер увеличивается), различия в их встречаемости у больных с OX (cм. табл. 1) указывают на то, что эта связь не абсолютная. В других исследованиях мы не нашли анализа влияния размера БСДПК на развитие ОХ, однако приведены данные о том, что наличие фиксированного камня БСДПК у больных с ОХ наблюдается достоверно чаще [6, 8].

\section{Выводы}

1. Факторами риска развития ОХ при ДОВЖП являются уровень общего билирубина 70 мкмоль/л и выше, тол- 
щина стенки ЖП 4 мм и больше, наличие отключенного ЖП, фиксированного камня БСДПК и размер БСДПК 15 мм и более.

2. Проявлениями ОХ при ДОВЖП являются гипертермия, наличие триады Шарко, лейкоцитоз $9 \times 10^{9}$ в 1 л и выше, количество палочкоядерных нейтрофилов 7\% и выше, гиперамилаземия.

\section{Подтверждение}

Финансирование. Личные средства авторов.

Информация об участии авторов. Мамонтов И. Н., Тамм Т. И.- концепция и дизайн исследования, сбор материала, статистическая обработка, анализ данных, написание текста; Крамаренко К. А., Белов С. Г. - анализ данных, сбор материала; Бардюк А. Я. - анализ данных, сбор материала; Захарчук А. Я., Непомнящий В. В., Шакалова Е. А., Решетняк О. М.- сбор материала.

Конфликт интересов. Авторы заявляют об отсутствии какого-либо конфликта интересов при подготовке данной статьи.

Согласие на публикацию. Все авторы прочитали и одобрили окончательный вариант рукописи. Все авторы дали согласие на публикацию этой рукописи.

\section{References}

1. Kimura Y, Takada T, Strasberg SM, Pitt HA, Gouma DJ, Garden OJ, et al. TG13 current terminology, etiology, and epidemiology of acute cholangitis and cholecystitis. J Hepatobiliary Pancreat Sci. 2013;20(1):8-23. doi 10.1007/s00534-012-0564-0.

2. Yamashita $Y$, Ueda $\mathrm{K}$, Abe H, Tamura T, Itonaga M, Yoshida $\mathrm{T}$, et al. Common Bile Duct Dilatation With Stones Indicates Requirement for Early Drainage in Patients With or Without Cholangitis. Gastroenterology Res. 2013;6(6):219-26. doi: 10.4021/gr587w.

3. Lan Cheong Wah D, Christophi C, Muralidharan V. Acute cholangitis: current concepts. ANZ J Surg. 2017;87(7-8):554-9. doi: 10.1111/ans.13981.

4. Sokal A, Sauvanet A, Fantin B, Lastours V. Acute cholangitis: Diagnosis and management. J Visc Surg. 2019. pii: S1878-7886(19)30067-0. doi: 10.1016/j.jviscsurg.2019.05.007.

5. Gigot JF, Leese T, Dereme T, Coutinho J, Castaing D, Bismuth H. Acute Cholangitis Multivariate Analysis of Risk Factors. Ann Surg. 1989;209(4):435-8. PMID: 2930289.

6. Csendes A, Diaz JC, Burdiles P, Maluenda F, Morales E. Risk factors and classification of acute suppurative cholangitis. Br. J. Surg. 1992;79(7):655-8. doi: 10.1002/bjs.1800790720.

7. Tsujinoa T, Sugitaa R, Yoshidaa H, Yagiokaa H, Kogurea H, Sasakia T, et al. Risk factors for acute suppurative cholangitis caused by bile duct stones. Eur J Gastroenterol Hepatol.2007;19(7):585-8. doi: 10.1097/ MEG.0b013e3281532b78.

8. Yeom DH, Oh HJ, Son YW, Kim TH. What Are the Risk Factors for Acute Suppurative Cholangitis Caused by Common Bile Duct Stones? Gut and Liver. 2010;4(3):363-7. doi: 10.5009/gn1.2010.4.3.363.

9. Yun-Sheng Qin, Qi-Yong Li, Fu-Chun Yang and Shu-Sen Zheng. Risk factors and incidence of acute pyogenic Cholangitis. HBPD International. 2012;11(6):650-4. doi: 10.1016/S1499-3872(12)60240-9.

10. Kiriyama S, Kozaka K, Takada T, Strasberg TM, Pitt HA, Gabata T, et al. Tokyo Guidelines 2018: diagnostic criteria and severity grading of acute cholangitis (with videos). J Hepatobiliary Pancreat Sci. 2018;25(1):1730. doi: $10.1002 /$ jhbp.512.

11. Miura F, Okamoto K, Takada T, Strasberg SM, Asbun HJ, Pitt HA, et al. Tokyo Guidelines 2018: initial management of acute biliary infectionand flowchart for acute cholangitis. J Hepatobiliary Pancreat Sci. 2018;25(1):31-40. doi: 10.1002/jhbp.509.

Надійшла 28.07.2019 